\title{
Knowledge, attitude and practices (KAP) towards COVID-19 and assessment of risks of infection by SARS-CoV-2 among the Bangladeshi population: An online cross sectional survey
}

\author{
Tasnima Haque \\ BIHS General Hospital, Dhaka, Bangladesh \\ Khondoker Moazzem Hossain \\ Biotechnology and Genetic Engineering Discipline, Khulna University, Khulna, Bangladesh \\ Md. Monzur Rahman Bhuiyan \\ AICHI Hospital Ltd. Dhaka, bangladesh \\ Sadia Afreen Ananna \\ BIHS General Hospital, Dhaka, Bangladesh \\ Md. Anower Hussain \\ Faculty of Public Health, Bangladesh University of Health Sciences \\ Mohammad Rafiqul Islam \\ Livestock Division, Bangladesh Agricultural Research Council, Dhaka, Bangladesh \\ Asif Ahmed \\ Biotechnology and Genetic Engineering Discipline, Khulna University, Khulna, Bangladesh
}

Mohammad Mahmudur Rahman ( $\square$ maahmud@gmail.com)

Dept. of Medical Biotechnology, Bangladesh University of Health Sciences, Dhaka, Bangladesh https://orcid.org/0000-0002-1211-8642

\section{Research Article}

Keywords: COVID-19, KAP, Bangladesh, Risk of Infection

Posted Date: September 16th, 2020

DOI: https://doi.org/10.21203/rs.3.rs-24562/v2

License: @ (i) This work is licensed under a Creative Commons Attribution 4.0 International License. Read Full License 


\section{Abstract}

The COVID19 pandemic has been transmitted worldwide rapidly. The best ways of preventing this virus are to know about and act accordingly. An online cross sectional survey was conducted to know the knowledge, attitude and practices towards COVID19 and to assess the risks of infections among Bangladeshi population. Among 2045 respondents, 54.87\% respondents kept good knowledge. Knowledge was significantly diverged across age, gender, education levels, residences, income groups, and marital status. Despite the knowledge, the attitude and practices of Bangladeshi people are not impressive. Among population, $32.08 \%$, and $44.30 \%$ people were in high, and in medium risk of infection respectively. Everybody is in risk. Reasons for the mediocre attitude and practices could be the poor knowledge, nonscientific and orthodox religious believe. Government and policy makers must consider these knowledge levels, attitude \& practices and the risk of infection assessment to implement productive interventions for preventing the COVID19.

\section{Introduction:}

COVID-19 is a contagious disease caused by newly identified coronavirus called severe acute respiratory syndrome coronavirus 2 [1].SARS-CoV-2 is first identified in Wuhan city of China in 2019 and has subsequently spread worldwide, ensuing in the current 2019-20 coronavirus pandemic[2,3].Till $8^{\text {th }}$ April 2020 , total cases of infection reach 1,536,652and death toll is 89,907 and the trend is going up, however, so far 340,349persons were recovered from COVID-19 [4].The lungs are the most affected organs in this disease as the virus enters via the enzyme called angiotensin converting enzyme 2 (ACE2) which is mostly profuse in the type II alveolar cells of the lungs [5].COVID19 patients may be asymptomatic or progress flu-like symptoms, with fever, dry cough, tiredness and shortness of breath[6]. Immediate medical attention is advised when severe symptoms including persistent chest pain or pressure, difficulty of breathing, confusion, and bluish face or lips arises [6]. Upper respiratory symptoms i.e. runny nose, sneezing or sore throat, vomiting, diarrhea, nausea, chest tightness, palpitations etc. have been witnessed in varying percentages [7,8].During March 2020, anosmia (loss of the sense of smell) was reported in some cases $[9,10]$. .In severe cases, the disease may develop pneumonia, multi organ failure and death [2,11], and requires onset to necessitating ventilations minimum for 8 days[12]. Gastrointestinal organs are also affected as ACE2 is expressed in the glandular cells of duodenal, gastric and rectal epithelium [13] as well as enterocytes of the small intestine and endothelial cells [14].

The SARS-CoV-2 belongs to the wide-ranging family of virus, coronavirus containing positive-sense single stranded RNA, and genetically close to bat coronavirus[15].Family of these viruses is known for developing human sickness including common cold to more severe diseases such as Sever Acute Respiratory Syndrome (SARS) and Middle East Respiratory Syndrome (MERS)[16].Persons infected with the virus need 2 to 14 days of incubation period to develop symptoms and $97.5 \%$ of patients express symptoms within 11.5 days [17].SARS-CoV-2 virus is predominantly spread between persons via respirational droplets from coughs and sneezes[12]. Studies demonstrated that this virus is live on copper for 4 to 18 hours, on cardboard for 24 to 55 hours, on plastics for 72 to 100 hours, stainless steels for 72 to 90 hours and in aerosol for three hours although the detection rates varies between surface materials types [18]. The virus also been isolated from human faeces, however, spread through it is being investigated [13]. Airborne characterizes of COVID-19 virus are not expressed yet[19].Infections can be prevented as per recommendations including repeated hand washing with soaps or alcohol based sanitizers, maintain social distances from others, covering coughs and sneezes to protect others, and avoiding hands away from mouth, nose, and eyes [11,20].

Public Health Emergency of International Concern (PHEIC) was declared on 30 January by the World Health Organization (WHO) for the coronavirus outbreak[21]and a pandemic on 11 March 2020. ${ }^{3}$ Bangladesh is one of the small but most populated (162.9 million and $8^{\text {th }}$ in the World)[22] and densely $\left(1,169\right.$ per $\left.\mathrm{km}^{2}\right)$ populated country [23].It is difficult to manage these large numbers of population especially in the pandemic conditions. The first coronavirus infection was found on 7 March 2020 and till $9^{\text {th }}$ April 2020, 330 people are infected and the deaths are seventeen[4]. Bangladesh Government has declared lockdown all over the countries except some emergency services instructing staying at home to avoid contacting with others, with the deferral of public transport, the closing of public spaces, close managing of communities, and isolation and care for infected people and suspected cases. To ensure the ultimate success, citizen's devotion to these control measures are important, which is generally affected by their knowledge, attitudes, and practices (KAP) to COVID-19 according to KAP theory[24,25]. Previous information and lessons from the world outbreak recommend that knowledge and attitudes towards contagious diseases are linked with level of sentiment among the population, which can further confuse efforts to stop the spread of the disease [26,27].To ease the pandemic controlling of COVID-19 in Bangladesh, there is a crucial need to understand the citizen's consciousness of COVID-19 at this complex situation. In this study, the knowledge, attitudes and practices (KAP) regarding COVID-19 outbreak among Bangladeshi population and its associated risks were investigated through online survey.

\section{Methodology:}

\section{Study Design, Setting and Participants}

The current study was designed to obtain the information regarding knowledge, attitude and practice towards COVID-19 and to assess the risk of infection through their daily practices. Online cross sectional questionnaires were used to obtain data. Questionnaires consisted of three parts. Demography, COVID-19 Knowledge Test (C19KT), and last part were to assess attitude and practices. Demographic variables included administrative divisions, age, gender, education, occupation, residence (urban/rural), monthly family income, and marital status. Data were obtained from all the eight administrative divisions of Bangladesh 
according to the proportion of population. The latest population census, 2011 was used to obtain the percentages of total people living in different Divisions of the country. According to 2011 census [23], the distributions of population and the collected data numbers was considered (Supplementary Table 01).

Online cross-sectional questionnaires were made available to all the social media users and provided extensively towards 10 volunteers. Volunteers and all the authors were associated regarding the online survey. The survey was launched on 14 March 2020 and closed on 30 March 2020. There was no specific exclusion criterion for participating in this survey except age. Anyone who were 16 years of old and above, were eligible to participate in this survey. A total of 2343participants responded. A total of 2045 filled questionnaire were selected for the data analysis.

\section{C19KT questionnaires and their assessment}

Forty questions (Supplementary Table 02) were included in the questionnaire to test the knowledge of the respondents. Every question had three possible answers, true, false and not sure; however, only one was the right answer. Every question carried one point. Respondents who scored more than 30 were identified as keeping "good knowledge" regarding COVID-19.

\section{Assessments of attitude and practices of respondents}

Attitude and practices were measured by respondent's regular lifestyles and knowledge regarding COVID-19 in this pandemic situation. To assess the attitude and practices of respondents towards COVID-19, twenty four questions (Supplementary Table 03) was used. Total 24 questions out of 22 had options to answer yes/no.

\section{Assessments of risk of infection}

Besides the answers of selected questions regarding attitude and practices, risk of infections was also measured. To perform this, 13 questions were selected from attitude and practice questions. The scores were calculated individually and cumulative scores were obtained for all. Based on the acquired scores out of total score thirty nine, the risk of infections was distributed accordingly (Supplementary Table 04 and Supplementary Table 05).Risk scores were then assessed using demographic classifications and C19KT to know the status of risk group.

\section{Handling of variables and processing for analysis}

Selected 2045 questionnaire were checked and re-checked several times carefully with the authors. Ages were divided into three groups, 16 to 30 years old, 31 to 55 years olds and 55+ years old. Education data ware ranged as up to HSC/Diploma and above HSC/Diploma; occupations data were ranged as unemployed (no job, house wife and students) and employed (Govt. job, NGO/private job and business); residences were divided as village/rural (village/rural and Upazila level) and urban (capital city, Divisional city and District towns). Non numeric data were coded with numbers for analysis.

\section{Statistical Analysis}

Multivariable linear regression and binary logistic regression analysis was used to identify factors associated with knowledge. Binary logistic regression analysis was conducted where Risk assessment was dependent variables. Regression coefficients ( $\beta$ ) and odds ratios (ORs) and their $95 \%$ confidence intervals (Cls) were used to quantify the associations between variables. Data were analyzed using SPSS version 26.0.

\section{Results:}

A total 2343 participants accomplished the online questionnaires. Data of 298participantsregarding unspecified answers, confusing responses and missing parameters were discarded. The final sample involved 2045 participants. Among the respondents, $512(25 \cdot 04 \%)$ were from Dhaka Division, the average age was 27.82 years of which(SD: 1.36, range 16-65); 1667 (81.52\%) were between 16 and 30 years old; 1085 (53.06\%) were man; 1057 (51.69\%) were graduates, $843(41 \cdot 22 \%)$ were students, 439 (21·47\%), 504 (24.65\%), and 575 (28.12\%) were from Capital city, Divisional cities, and District towns, respectively,838 (40.98\%) were from middle income group whose monthly family income were between Taka 25,000/00 to60,000/00, and 1320 (64.55\%) never married (See Table 01).

Table 01: Demographic classification of respondents on the basis of overall and good knowledge on 
Page 4/16 


\begin{tabular}{|c|c|c|c|c|c|}
\hline & \multicolumn{2}{|l|}{ Overall } & \multicolumn{3}{|c|}{ Good Knowledge } \\
\hline & Number & Percentages & Number & $\begin{array}{l}\text { Percentages } \\
\text { of total respondents }\end{array}$ & $\begin{array}{l}\text { Percentages } \\
\text { (within GKP) }\end{array}$ \\
\hline Respondents & 2045 & $100 \%$ & 1122 & $54 \cdot 87 \%$ & $100 \%$ \\
\hline \multicolumn{6}{|l|}{ Divisional classification } \\
\hline Barishal & 117 & $5 \cdot 72 \%$ & 77 & $3 \cdot 77 \%$ & $6 \cdot 86 \%$ \\
\hline Chittagong & 400 & $19 \cdot 56 \%$ & 209 & $10 \cdot 22 \%$ & $18 \cdot 63 \%$ \\
\hline Dhaka & 512 & $25 \cdot 04 \%$ & 309 & $15 \cdot 11 \%$ & $27 \cdot 54 \%$ \\
\hline Khulna & 240 & $11 \cdot 74 \%$ & 132 & $6 \cdot 45 \%$ & $11 \cdot 76 \%$ \\
\hline Mymensingh & 120 & $5 \cdot 87 \%$ & 30 & $1 \cdot 47 \%$ & $2 \cdot 67 \%$ \\
\hline Rajshahi & 269 & $13 \cdot 15 \%$ & 188 & $9 \cdot 19 \%$ & $16 \cdot 76 \%$ \\
\hline Rangpur & 252 & $12 \cdot 32 \%$ & 132 & $6 \cdot 45 \%$ & $11 \cdot 76 \%$ \\
\hline Sylhet & 135 & $6 \cdot 60 \%$ & 45 & $2 \cdot 20 \%$ & $4 \cdot 01 \%$ \\
\hline Age (Mean St. Dev.) & $27 \cdot 82$ & $1 \cdot 36$ & $27 \cdot 24$ & $6 \cdot 61$ & NA \\
\hline \multicolumn{6}{|l|}{ Age Range } \\
\hline 16 to 30 Years Old & 1667 & $81 \cdot 52 \%$ & 892 & $43 \cdot 62 \%$ & $79 \cdot 50 \%$ \\
\hline 31 to 55 Years Old & 365 & $17 \cdot 85 \%$ & 223 & $10 \cdot 90 \%$ & $19 \cdot 88 \%$ \\
\hline $55+$ Years Old & 13 & $0.64 \%$ & 7 & $0 \cdot 34 \%$ & $0 \cdot 62 \%$ \\
\hline \multicolumn{6}{|l|}{ Gender } \\
\hline Male & 1085 & $53.06 \%$ & 643 & $31 \cdot 44 \%$ & $57 \cdot 31 \%$ \\
\hline Female & 960 & $46 \cdot 94 \%$ & 479 & $23 \cdot 42 \%$ & $42 \cdot 69 \%$ \\
\hline \multicolumn{6}{|l|}{ Education } \\
\hline Class Five & 6 & $0 \cdot 29 \%$ & 4 & $0 \cdot 20 \%$ & $0 \cdot 36 \%$ \\
\hline SSC & 68 & $3 \cdot 33 \%$ & 0 & $0 \cdot 00 \%$ & $0 \cdot 00 \%$ \\
\hline HSC/Diploma & 990 & $48 \cdot 41 \%$ & 155 & $7 \cdot 58 \%$ & $13 \cdot 81 \%$ \\
\hline Graduates & 1057 & $51 \cdot 69 \%$ & 590 & $28.85 \%$ & $52 \cdot 58 \%$ \\
\hline Masters & 576 & $28 \cdot 17 \%$ & 331 & $16 \cdot 19 \%$ & $29 \cdot 50 \%$ \\
\hline Doctoral and over & 52 & $2 \cdot 54 \%$ & 42 & $2 \cdot 05 \%$ & $3 \cdot 74 \%$ \\
\hline \multicolumn{6}{|l|}{ Education Range } \\
\hline Up to HSC/Diploma & 360 & $17 \cdot 60 \%$ & 159 & $7 \cdot 78 \%$ & $14 \cdot 17 \%$ \\
\hline Above HSV/Diploma & 1685 & $82 \cdot 40 \%$ & 963 & $47 \cdot 09 \%$ & $85 \cdot 83 \%$ \\
\hline \multicolumn{6}{|l|}{ Occupation } \\
\hline Students & 843 & $41 \cdot 22 \%$ & 407 & $19 \cdot 90 \%$ & $36 \cdot 27 \%$ \\
\hline House Wife & 101 & $4 \cdot 94 \%$ & 41 & $2 \cdot 00 \%$ & $3 \cdot 65 \%$ \\
\hline Govt· Job & 211 & $10 \cdot 32 \%$ & 126 & $6 \cdot 16 \%$ & $11 \cdot 23 \%$ \\
\hline Private Job & 607 & $29 \cdot 68 \%$ & 365 & $17 \cdot 85 \%$ & $32 \cdot 53 \%$ \\
\hline Business & 96 & $4.69 \%$ & 25 & $1 \cdot 22 \%$ & $2 \cdot 23 \%$ \\
\hline No Jobs & 187 & $9 \cdot 14 \%$ & 158 & $7 \cdot 73 \%$ & $14.08 \%$ \\
\hline \multicolumn{6}{|l|}{ Residence } \\
\hline Capital City & 439 & $21 \cdot 47 \%$ & 236 & $11 \cdot 54 \%$ & $21 \cdot 03 \%$ \\
\hline Divisional City & 504 & $24 \cdot 65 \%$ & 228 & $11 \cdot 15 \%$ & $20 \cdot 32 \%$ \\
\hline District Town & 575 & $28 \cdot 12 \%$ & 311 & $15 \cdot 21 \%$ & $27 \cdot 72 \%$ \\
\hline Upazila (Sub District) Town & 266 & $13 \cdot 01 \%$ & 155 & $7 \cdot 58 \%$ & $13 \cdot 81 \%$ \\
\hline Village or Rural & 261 & $12 \cdot 76 \%$ & 192 & $9 \cdot 39 \%$ & $17 \cdot 11 \%$ \\
\hline
\end{tabular}


Monthly Family Income

\begin{tabular}{llllll}
\hline Less than Taka 25,000/00 & 571 & $27 \cdot 92 \%$ & 283 & $13 \cdot 84 \%$ & $25 \cdot 22 \%$ \\
\hline Taka 25,000/00 to Taka 60,000/00 & 838 & $40 \cdot 98 \%$ & 462 & $22 \cdot 59 \%$ & $41 \cdot 18 \%$ \\
\hline Over Taka 60000 & 636 & $31 \cdot 10 \%$ & 377 & $18 \cdot 44 \%$ & $33 \cdot 60 \%$ \\
\hline Marital Status & & & & & \\
\hline Never Married & 1320 & $64 \cdot 55 \%$ & 704 & $34 \cdot 43 \%$ & $62 \cdot 75 \%$ \\
\hline Ever Married & 725 & $35 \cdot 45 \%$ & 418 & $20 \cdot 44 \%$ & $37 \cdot 25 \%$ \\
\hline C19KT Score (Mean, SD) & 30.41 & 3.55 & NA & NA & NA \\
\hline C19KT & & & & & NA \\
\hline Poor Knowledge & 923 & $45 \cdot 13 \%$ & NA & NA & NA
\end{tabular}

Demographic classification of all respondents and the good knowledge keeping respondents. COVID19= Coronavirus disease 19, GKP= Good Knowledge Person, C19KT=COVID19 Knowledge Test.

Among the 40 questions on the COVID-19,those answered 30+,was considered them having good knowledge. Among 2045 respondents, 1122 (54.87\%)

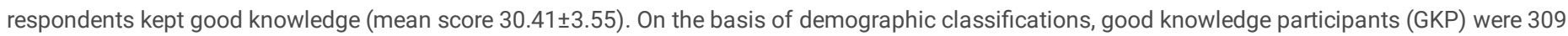
(15.11\%) among total respondents and $27 \cdot 54 \%$ of GKP were from Dhaka Division, average age were $27 \cdot 24 \pm 6.61,643(31 \cdot 44 \%$ of overall and $57 \cdot 31 \%$ of GKP) were man, 590 ( $28.85 \%$ of overall and $52 \cdot 58 \%$ of GKP) were graduates. For detail demographic classification of good knowledge towards COVID19 please see Table 01.

The average C19KT score suggested that most of the participants had good knowledge on COVID-19. Multivariable linear regression and binary logistic regression analysis demonstrated that C19KT scores significantly diverged across age, gender, education level, residence, monthly family income and marital status. However, occupation did not show significant association. Detail association of demographic characters towards knowledge was shown in Table 02.

Table 02: Multivariable linear and binary logistic regressions regarding poor knowledge factors associated with COVID-19

\begin{tabular}{|c|c|c|c|c|c|c|}
\hline \multirow[b]{2}{*}{ Variables } & \multicolumn{3}{|c|}{ Multivariable linear regression } & \multicolumn{3}{|c|}{ Binary logistic regression } \\
\hline & $\begin{array}{l}\text { Coefficient } \\
(\beta)\end{array}$ & $\begin{array}{l}\text { Standard } \\
\text { error }\end{array}$ & $\mathbf{t}$ & $\begin{array}{l}\text { Odds Ratio } \\
\text { (OR) }\end{array}$ & $\begin{array}{l}95 \% \text { Confidence } \\
\text { Intervals }\end{array}$ & $\mathbf{P}$ \\
\hline Age Range ( 16 to 13 years old vs $31+$ years old) & $-0 \cdot 128$ & 0.034 & $-4 \cdot 813$ & 0.497 & $0 \cdot 372-0 \cdot 663$ & $<0.001$ \\
\hline Gender (male vs female) & -0.097 & 0.023 & $-4 \cdot 269$ & 0.666 & $0 \cdot 551-0 \cdot 805$ & $<0.001$ \\
\hline $\begin{array}{l}\text { Education Range (up to HSC/Diploma vs above } \\
\text { HSC/Diploma) }\end{array}$ & $0 \cdot 116$ & 0.030 & $5 \cdot 016$ & 1.921 & $1 \cdot 485-2 \cdot 486$ & $<0.001$ \\
\hline Occupation (Non employed vs Employed) & 0.012 & 0.026 & $0 \cdot 466$ & $1 \cdot 054$ & $0 \cdot 848-1 \cdot 310$ & 0.636 \\
\hline Residence (Village/Rural and Upazila vs Urban) & $-0 \cdot 162$ & 0.026 & $-7 \cdot 017$ & $0 \cdot 450$ & $0 \cdot 358-0 \cdot 566$ & $<0.001$ \\
\hline Monthly Family Income (Low and medium vs High) & 0.051 & 0.025 & $2 \cdot 219$ & $1 \cdot 251$ & $1 \cdot 018-1 \cdot 536$ & 0.033 \\
\hline Marital Status (Never married vs Ever Married) & $0 \cdot 122$ & 0.028 & $4 \cdot 483$ & $1 \cdot 720$ & $1 \cdot 351-2 \cdot 189$ & $<0.001$ \\
\hline
\end{tabular}

The reference category of the both analysis was the first category of each independent variable. C10KT score was the dependent variables in both cases (poor knowledge was reference).

The most of the respondents frequently washed their hands (95.45\%) in this pandemic conditions. Around 1545 (75.55\%) respondents wore masks when going out, however, the patterns of cleaning of used masks were not impressive. Around $8 \cdot 17 \%$ respondents did not use masks even in this alarming situations. Among 686 respondents, 33.55\% respondents cleaned their mask every day. Rest of the respondents wore from 2 to 60 days without proper cleaning. The rates of disposing used cloths and shoes after returning from outside were $68 \cdot 12 \%$ and $29 \cdot 63 \%$, respectively, suggesting people are less concern 
about transmission of viruses through dresses and shoes. About $87.97 \%$ respondents maintained social distances and $67.73 \%$ were not spending times with friends and colleagues after work or classes. In leisure time, among 2045 respondents, 1400 (68.46\%) and 295 (14.43\%) were not going to roadside shops for tea/coffee and snacks, respectively. Though, in average $4.75 \pm 2.61$ days in a week, respondents were taking roadside snacks. In total 661 (32.32\%)

respondents dealt with sick people. Very few participants visited corona infected areas (2.98\%) and met with the people who came from corona infected areas (1.91\%). Ten respondents reported that their family members were affected with coronavirus. Among common practices, $62 \cdot 15 \%$ participants sneezed between elbows, and $62.93 \%$ did not touch mouth, nose or eyes with dirty hands (Table 03).

Table 03: Attitude and practices of respondents based on overall and good knowledge 


\begin{tabular}{|c|c|c|c|c|c|}
\hline & & \multicolumn{2}{|c|}{ Overall $(n=2045)$} & \multicolumn{2}{|c|}{ Good Knowledge $(n=1122)$} \\
\hline & & $\begin{array}{l}\text { Number/ } \\
\text { Mean }\end{array}$ & $\begin{array}{l}\text { Percentages/ } \\
\text { SD }\end{array}$ & $\begin{array}{l}\text { Number/ } \\
\text { Mean }\end{array}$ & $\begin{array}{l}\text { Percentages/ } \\
\text { SD }\end{array}$ \\
\hline \multirow[t]{2}{*}{ Frequently washed hands with soap or sanitizer } & No & 93 & $4 \cdot 55 \%$ & 21 & $1 \cdot 87 \%$ \\
\hline & Yes & 1952 & $95 \cdot 45 \%$ & 1101 & $98 \cdot 13 \%$ \\
\hline \multirow[t]{2}{*}{ Regular use of mask } & No & 500 & $24 \cdot 45 \%$ & 231 & $20 \cdot 59 \%$ \\
\hline & Yes & 1545 & $75 \cdot 55 \%$ & 891 & $79 \cdot 41 \%$ \\
\hline \multirow[t]{15}{*}{ Number of days used mask without proper cleaning } & Mean and SD. & $4 \cdot 61$ & $8 \cdot 43$ & $1 \cdot 59$ & $0 \cdot 32$ \\
\hline & Do not use mask & 167 & $8 \cdot 17 \%$ & 32 & $2 \cdot 85 \%$ \\
\hline & 1 Day & 686 & $33 \cdot 55 \%$ & 595 & $53.03 \%$ \\
\hline & 2 Days & 314 & $15 \cdot 35 \%$ & 298 & $26 \cdot 56 \%$ \\
\hline & 3 Days & 249 & $12 \cdot 18 \%$ & 197 & $17 \cdot 56 \%$ \\
\hline & 4 Days & 36 & $1 \cdot 76 \%$ & 0 & $0.00 \%$ \\
\hline & 5 Days & 82 & $4 \cdot 01 \%$ & 0 & $0.00 \%$ \\
\hline & 6 Days & 6 & $0 \cdot 29 \%$ & 0 & $0.00 \%$ \\
\hline & 7 Days & 368 & $18 \cdot 00 \%$ & 0 & $0.00 \%$ \\
\hline & 10 Days & 45 & $2 \cdot 20 \%$ & 0 & $0.00 \%$ \\
\hline & 12 Days & 2 & $0 \cdot 10 \%$ & 0 & $0.00 \%$ \\
\hline & 15 Days & 12 & $0.57 \%$ & 0 & $0.00 \%$ \\
\hline & 20 Days & 6 & $0 \cdot 29 \%$ & 0 & $0.00 \%$ \\
\hline & 30 Days & 42 & $2 \cdot 05 \%$ & 0 & $0.00 \%$ \\
\hline & 60 Days & 30 & $1 \cdot 47 \%$ & 0 & $0.00 \%$ \\
\hline \multirow{4}{*}{$\begin{array}{l}\text { What usually you do with your dress when you come } \\
\text { from outside? }\end{array}$} & Do not wash or & 652 & $31 \cdot 88 \%$ & 313 & $27 \cdot 90 \%$ \\
\hline & Wear next day & & & & \\
\hline & Washed or & 1393 & $68 \cdot 12 \%$ & 809 & $72 \cdot 10 \%$ \\
\hline & Left for wash & & & & \\
\hline \multirow{4}{*}{$\begin{array}{l}\text { What usually you do with your shoes when you come } \\
\text { from outside? }\end{array}$} & Do not wash or & 1439 & $70 \cdot 37 \%$ & 836 & $74.51 \%$ \\
\hline & Wear next day & & & & \\
\hline & Washed or & 606 & $29 \cdot 63 \%$ & 286 & $25 \cdot 49 \%$ \\
\hline & Left for wash & & & & \\
\hline \multirow[t]{2}{*}{ Generally you use mass transport } & No & 789 & $38 \cdot 58 \%$ & 453 & $40 \cdot 37 \%$ \\
\hline & Yes & 1256 & $61 \cdot 42 \%$ & 669 & $59 \cdot 63 \%$ \\
\hline \multirow[t]{2}{*}{ Usually spend times with friends regularly } & No & 1385 & $67 \cdot 73 \%$ & 831 & $74 \cdot 06 \%$ \\
\hline & Yes & 660 & $32 \cdot 27 \%$ & 291 & $25 \cdot 94 \%$ \\
\hline \multirow[t]{2}{*}{ Maintained social distances } & No & 246 & $12 \cdot 03 \%$ & 167 & $14 \cdot 88 \%$ \\
\hline & Yes & 1799 & $87 \cdot 97 \%$ & 955 & $85 \cdot 12 \%$ \\
\hline \multirow[t]{2}{*}{ Sneezed between elbows } & No & 774 & $37 \cdot 85 \%$ & 324 & $28 \cdot 88 \%$ \\
\hline & Yes & 1271 & $62 \cdot 15 \%$ & 798 & $71 \cdot 12 \%$ \\
\hline \multirow[t]{2}{*}{ Frequently touched mouth or eyes or nose } & No & 758 & $37 \cdot 07 \%$ & 671 & $59 \cdot 80 \%$ \\
\hline & Yes & 1287 & $62 \cdot 93 \%$ & 451 & $40 \cdot 20 \%$ \\
\hline \multirow[t]{2}{*}{ Usually drink tea/coffee from road side tea shops } & No & 1400 & $68 \cdot 46 \%$ & 818 & $72 \cdot 91 \%$ \\
\hline & Yes & 645 & $31 \cdot 54034$ & 304 & $27 \cdot 09 \%$ \\
\hline \multirow[t]{2}{*}{ Days in a week usually eat roadside snacks } & Mean and SD & $4 \cdot 75$ & $2 \cdot 61$ & $3 \cdot 79$ & $1 \cdot 22 \%$ \\
\hline & $\begin{array}{l}\text { Do not eat snacks from } \\
\text { roadside }\end{array}$ & 295 & $14 \cdot 43 \%$ & 248 & $22 \cdot 10 \%$ \\
\hline
\end{tabular}




\begin{tabular}{|c|c|c|c|c|c|}
\hline & 1 & 30 & $1 \cdot 47 \%$ & 29 & $2 \cdot 58 \%$ \\
\hline & 2 & 98 & $4 \cdot 79 \%$ & 67 & $5 \cdot 97 \%$ \\
\hline & 3 & 211 & $10 \cdot 32 \%$ & 187 & $16 \cdot 67 \%$ \\
\hline & 4 & 193 & $9 \cdot 44 \%$ & 125 & $11 \cdot 14 \%$ \\
\hline & 5 & 211 & $10 \cdot 32 \%$ & 118 & $10 \cdot 52 \%$ \\
\hline & 6 & 421 & $20 \cdot 57 \%$ & 211 & $18 \cdot 81 \%$ \\
\hline & 7 & 586 & $28 \cdot 66 \%$ & 137 & $12 \cdot 21 \%$ \\
\hline \multirow[t]{2}{*}{ Regularly clean work or home or classroom table } & No & 536 & $26 \cdot 21 \%$ & 293 & $26 \cdot 11 \%$ \\
\hline & Yes & 1509 & $73 \cdot 79 \%$ & 829 & $73 \cdot 89 \%$ \\
\hline \multirow[t]{2}{*}{ Usually clean mobile with sanitizer } & No & 1227 & $60 \cdot 00 \%$ & 608 & $54 \cdot 19 \%$ \\
\hline & Yes & 818 & $40 \cdot 00 \%$ & 514 & $45 \cdot 81 \%$ \\
\hline \multirow[t]{2}{*}{ Usually touch mobile phone with unwashed hands } & No & 1040 & $50 \cdot 86 \%$ & 583 & $51 \cdot 96 \%$ \\
\hline & Yes & 1005 & $49 \cdot 14 \%$ & 539 & $48 \cdot 04 \%$ \\
\hline \multirow[t]{2}{*}{ Regularly deal with sick people or health worker } & No & 1384 & $67 \cdot 68 \%$ & 718 & $63.99 \%$ \\
\hline & Yes & 661 & $32 \cdot 32 \%$ & 404 & $36 \cdot 01 \%$ \\
\hline \multirow[t]{2}{*}{ Usually share food or water pot with others } & No & 1448 & $70 \cdot 81 \%$ & 778 & $69 \cdot 34 \%$ \\
\hline & Yes & 597 & $29 \cdot 19 \%$ & 344 & $30 \cdot 66 \%$ \\
\hline \multirow{2}{*}{$\begin{array}{l}\text { Often eat half or semi cooked fish, meat, eggs or } \\
\text { vegetables }\end{array}$} & No & 1720 & $84 \cdot 11 \%$ & 953 & $84 \cdot 94 \%$ \\
\hline & Yes & 325 & $15 \cdot 89 \%$ & 169 & $15 \cdot 06 \%$ \\
\hline \multirow[t]{2}{*}{ Recently visited corona virus infected area } & No & 1984 & $97 \cdot 02 \%$ & 1080 & $96 \cdot 26 \%$ \\
\hline & Yes & 61 & $2 \cdot 98 \%$ & 16 & $1 \cdot 43 \%$ \\
\hline \multirow{2}{*}{$\begin{array}{l}\text { Recently met with people came from abroad (corona } \\
\text { affected area) }\end{array}$} & No & 2006 & $98 \cdot 09 \%$ & 1106 & $98 \cdot 57 \%$ \\
\hline & Yes & 39 & $1 \cdot 91 \%$ & 16 & $1 \cdot 43 \%$ \\
\hline \multirow{2}{*}{$\begin{array}{l}\text { COVID-19 symptoms at your work places or near work } \\
\text { place }\end{array}$} & No & 1874 & $91 \cdot 64 \%$ & 1049 & $93 \cdot 49 \%$ \\
\hline & Yes & 171 & $8 \cdot 36 \%$ & 73 & $6 \cdot 51 \%$ \\
\hline \multirow[t]{2}{*}{ Family member(s) caught corona infection } & No & 2035 & $99 \cdot 51 \%$ & 1118 & $99 \cdot 64 \%$ \\
\hline & Yes & 10 & $0.49 \%$ & 4 & $0.36 \%$ \\
\hline \multirow[t]{2}{*}{ Quarantine facilities near your home or workplace } & No & 1714 & $83 \cdot 81 \%$ & 989 & $88 \cdot 15 \%$ \\
\hline & Yes & 331 & $16 \cdot 19 \%$ & 138 & $12 \cdot 30 \%$ \\
\hline \multirow[t]{2}{*}{ Home or work places clean everyday with sanitizer } & No & 146 & $7 \cdot 14 \%$ & 34 & $3.03 \%$ \\
\hline & Yes & 1899 & $92 \cdot 86 \%$ & 1088 & $96.97 \%$ \\
\hline
\end{tabular}

Attitude and practices were measured according to the answer given by the respondents. In this table, attitude and practices were compared between all respondents and good knowledge respondents to assess the association of good knowledge and practices.

Assessments of risks of infection were analyzed among all the respondents based on the thirteen selected attitude and practices questions. According to the risks categories among all respondents, 373 (18.24\%) did belong to low risk category with average risk score 8.05 $\pm 1 \cdot 18,906(44 \cdot 30 \%)$ were in medium risk

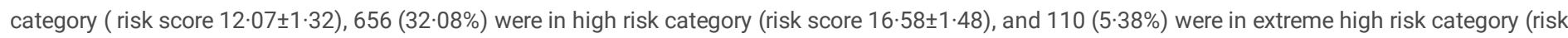
score $21 \cdot 77 \pm 1 \cdot 45)$. However, no respondents were found risk free and this is the dangerous characteristics of COVID-19.Among extreme high risk (HER) category, 46 ( $41 \cdot 82 \%$ of HER and $2 \cdot 25 \%$ of all) were from Dhaka Division, 87 (79.09\% of HER and $4.25 \%$ of all) were in age group between 16 to 30 years old, 63 (57.27\% of HER and $3.08 \%$ of all) were female, 98 (89.09\% of HER and $4.79 \%$ of all) had education levels above HSC/Diploma, 51 (46.36\% of HER and $2 \cdot 49 \%$ of all) were private job holder, 39 (35.45\% of HER and $1.91 \%$ of all) lives in the Capital city, 47 ( $42 \cdot 73 \%$ of HER and $2 \cdot 30 \%$ of all) were from high income group, 58 (52.73\% of HER and $2 \cdot 84 \%$ of all) never married and 75 (68.18\% of HER and $3.67 \%$ of all) kept good C19KT score. Respondents from Barishal Division were not in extreme high risk. In high risk (HR) group, trends were like as HER except residence, income group and C19KT score. Detail demographic characteristics of $\mathrm{n}$ of infection are shown in Table 04. 
Table 04: Risks of infections according to demographic classification

Page 10/16 


\begin{tabular}{|c|c|c|c|c|c|c|c|c|c|c|c|}
\hline & \multicolumn{3}{|c|}{ Extreme High Risk (HER) } & \multicolumn{3}{|c|}{ High Risk (HR) } & \multicolumn{3}{|c|}{ Medium Risk (MR) } & \multicolumn{2}{|c|}{ Low Risk (LR) } \\
\hline & Number & $\begin{array}{l}\text { Percentages } \\
\text { of total } \\
\text { Population }\end{array}$ & $\begin{array}{l}\text { Percentage } \\
\text { (within } \\
\text { HER) }\end{array}$ & Number & $\begin{array}{l}\text { Percentages } \\
\text { of total } \\
\text { Population }\end{array}$ & $\begin{array}{l}\text { Percentage } \\
\text { (within HR) }\end{array}$ & Number & $\begin{array}{l}\text { Percentages } \\
\text { of total } \\
\text { Population }\end{array}$ & $\begin{array}{l}\text { Percentage } \\
\text { (within } \\
\text { MR) }\end{array}$ & Number & $\begin{array}{l}\mathrm{Pe} \\
\text { of } \\
\mathrm{Po}\end{array}$ \\
\hline Respondents & 110 & $5 \cdot 38 \%$ & $100 \cdot 00 \%$ & 656 & $32.08 \%$ & $100 \cdot 00 \%$ & 906 & $44 \cdot 30 \%$ & $100 \cdot 00 \%$ & 373 & 18 \\
\hline $\begin{array}{l}\text { Risk Score } \\
\text { (Mean, SD) }\end{array}$ & $21 \cdot 77$ & $1 \cdot 45$ & NA & $16 \cdot 58$ & $1 \cdot 48$ & NA & $12 \cdot 07$ & $1 \cdot 32$ & NA & $8 \cdot 05$ & $1 \cdot 1$ \\
\hline \multicolumn{12}{|l|}{$\begin{array}{l}\text { Divisional } \\
\text { classification }\end{array}$} \\
\hline Barishal & 0 & $0.00 \%$ & $0.00 \%$ & 24 & $1 \cdot 17 \%$ & $3 \cdot 66 \%$ & 53 & $2 \cdot 59 \%$ & $5 \cdot 85 \%$ & 40 & $1 \cdot \mathrm{c}$ \\
\hline Chittagong & 6 & $0 \cdot 29 \%$ & $5 \cdot 45 \%$ & 115 & $5 \cdot 62 \%$ & $17 \cdot 53 \%$ & 220 & $10 \cdot 76 \%$ & $24 \cdot 28 \%$ & 59 & $2 \cdot \varepsilon$ \\
\hline Dhaka & 46 & $2 \cdot 25 \%$ & $41 \cdot 82 \%$ & 169 & $8 \cdot 26 \%$ & $25 \cdot 76 \%$ & 230 & $11 \cdot 25 \%$ & $25 \cdot 39 \%$ & 67 & $3 \%$ \\
\hline Khulna & 19 & $0.93 \%$ & $17 \cdot 27 \%$ & 79 & $3 \cdot 86 \%$ & $12 \cdot 04 \%$ & 99 & $4 \cdot 84 \%$ & $10 \cdot 93 \%$ & 43 & $2 \cdot 1$ \\
\hline Mymensingh & 7 & $0 \cdot 34 \%$ & $6 \cdot 36 \%$ & 50 & $2 \cdot 44 \%$ & $7 \cdot 62 \%$ & 45 & $2 \cdot 20 \%$ & $4.97 \%$ & 18 & $0 \cdot \xi$ \\
\hline Rajshahi & 19 & $0.93 \%$ & $17 \cdot 27 \%$ & 99 & $4 \cdot 84 \%$ & $15 \cdot 09 \%$ & 106 & $5 \cdot 18 \%$ & $11 \cdot 70 \%$ & 45 & $2 \cdot$ \\
\hline Rangpur & 12 & $0.59 \%$ & $10 \cdot 91 \%$ & 90 & $4 \cdot 40 \%$ & $13 \cdot 72 \%$ & 107 & $5 \cdot 23 \%$ & $11 \cdot 81 \%$ & 43 & $2 \cdot 1$ \\
\hline Sylhet & 1 & $0.05 \%$ & $0.91 \%$ & 30 & $1 \cdot 47 \%$ & $4 \cdot 57 \%$ & 46 & $2 \cdot 25 \%$ & $5 \cdot 08 \%$ & 58 & $2 \cdot \varepsilon$ \\
\hline $\begin{array}{l}\text { Age (Mean, } \\
\text { SD) }\end{array}$ & $28 \cdot 54$ & $7 \cdot 65 \%$ & NA & $27 \cdot 14$ & $6 \cdot 69 \%$ & NA & $26 \cdot 43$ & $6 \cdot 48$ & NA & $26 \cdot 49$ & $6 \cdot \varepsilon$ \\
\hline \multicolumn{12}{|l|}{ Age Range } \\
\hline $\begin{array}{l}16 \text { to } 30 \\
\text { Years Old }\end{array}$ & 87 & $4 \cdot 25 \%$ & $79 \cdot 09 \%$ & 506 & $24 \cdot 74 \%$ & $77 \cdot 13 \%$ & 756 & $36 \cdot 97 \%$ & $83 \cdot 44 \%$ & 318 & 15 \\
\hline $\begin{array}{l}31 \text { to } 55 \\
\text { Years Old }\end{array}$ & 18 & $0 \cdot 88 \%$ & $16 \cdot 36 \%$ & 143 & $6.99 \%$ & $21 \cdot 80 \%$ & 149 & $7 \cdot 29 \%$ & $16 \cdot 45 \%$ & 55 & $2 \cdot t$ \\
\hline $55+$ Years Old & 5 & $0 \cdot 24 \%$ & $4 \cdot 55 \%$ & 7 & $0.34 \%$ & $1 \cdot 07 \%$ & 1 & $0.05 \%$ & $0 \cdot 11 \%$ & 0 & $0 \cdot($ \\
\hline \multicolumn{12}{|l|}{ Gender } \\
\hline Male & 47 & $2 \cdot 30 \%$ & $42 \cdot 73 \%$ & 232 & $11 \cdot 34 \%$ & $35 \cdot 37 \%$ & 553 & $27 \cdot 04 \%$ & $61 \cdot 04 \%$ & 253 & 12 \\
\hline Female & 63 & $3.08 \%$ & $57 \cdot 27 \%$ & 424 & $20 \cdot 73 \%$ & $64 \cdot 63 \%$ & 353 & $17 \cdot 26 \%$ & $38.96 \%$ & 120 & $5 \cdot \xi$ \\
\hline \multicolumn{12}{|l|}{ Education } \\
\hline Class Five & 0 & $0.00 \%$ & $0.00 \%$ & 2 & $0 \cdot 10 \%$ & $0 \cdot 30 \%$ & 3 & $0 \cdot 15 \%$ & $0 \cdot 33 \%$ & 1 & $0 \cdot($ \\
\hline SSC & 6 & $0 \cdot 29 \%$ & $5 \cdot 45 \%$ & 39 & $1 \cdot 91 \%$ & $5.95 \%$ & 18 & $0 \cdot 88 \%$ & $1.99 \%$ & 1 & $0 \cdot c$ \\
\hline HSC/Diploma & 6 & $0 \cdot 29 \%$ & $5 \cdot 45 \%$ & 48 & $2 \cdot 35 \%$ & $7 \cdot 32 \%$ & 175 & $8 \cdot 56 \%$ & $19 \cdot 32 \%$ & 61 & $2 \cdot c$ \\
\hline Graduates & 69 & $3 \cdot 37 \%$ & $62 \cdot 73 \%$ & 340 & $16 \cdot 63 \%$ & $51 \cdot 83 \%$ & 434 & $21 \cdot 22 \%$ & $47 \cdot 90 \%$ & 214 & 10 \\
\hline Masters & 28 & $1 \cdot 37 \%$ & $25 \cdot 45 \%$ & 220 & $10 \cdot 76 \%$ & $33 \cdot 54 \%$ & 255 & $12 \cdot 47 \%$ & $28 \cdot 15 \%$ & 73 & $3 \cdot 5$ \\
\hline $\begin{array}{l}\text { Doctoral and } \\
\text { over }\end{array}$ & 1 & $0.05 \%$ & $0.91 \%$ & 7 & $0 \cdot 34 \%$ & $1 \cdot 07 \%$ & 21 & $1 \cdot 03 \%$ & $2 \cdot 32 \%$ & 23 & $1 \cdot 1$ \\
\hline \multicolumn{12}{|l|}{$\begin{array}{l}\text { Education } \\
\text { range }\end{array}$} \\
\hline $\begin{array}{l}\text { Up to } \\
\text { HSC/Diploma }\end{array}$ & 12 & $0 \cdot 59 \%$ & $10 \cdot 91 \%$ & 89 & $4 \cdot 35 \%$ & $13 \cdot 57 \%$ & 196 & $9 \cdot 58 \%$ & $21 \cdot 63 \%$ & 63 & $3 \cdot($ \\
\hline $\begin{array}{l}\text { Above } \\
\text { HSC/Diploma }\end{array}$ & 98 & $4 \cdot 79 \%$ & $89.09 \%$ & 567 & $27 \cdot 73 \%$ & $86 \cdot 43 \%$ & 710 & $34 \cdot 72 \%$ & $78 \cdot 37 \%$ & 310 & 15 \\
\hline \multicolumn{12}{|l|}{ Occupation } \\
\hline No Jobs & 4 & $0 \cdot 20 \%$ & $3 \cdot 64 \%$ & 36 & $1 \cdot 76 \%$ & $5 \cdot 49 \%$ & 95 & $4 \cdot 65 \%$ & $10 \cdot 49 \%$ & 52 & $2 \cdot 5$ \\
\hline House Wife & 6 & $0 \cdot 29 \%$ & $5 \cdot 45 \%$ & 45 & $2 \cdot 20 \%$ & $6 \cdot 86 \%$ & 41 & $2 \cdot 00 \%$ & $4.53 \%$ & 9 & 0.4 \\
\hline Students & 31 & $1 \cdot 52 \%$ & $28 \cdot 18 \%$ & 227 & $11 \cdot 10 \%$ & $34 \cdot 60 \%$ & 410 & $20.05 \%$ & $45 \cdot 25 \%$ & 175 & $8 \cdot !$ \\
\hline Govt· Job & 15 & $0 \cdot 73 \%$ & $13 \cdot 64 \%$ & 72 & $3.52 \%$ & $10 \cdot 98 \%$ & 54 & $2 \cdot 64 \%$ & $5.96 \%$ & 70 & $3 \cdot 4$ \\
\hline Private Job & 51 & $2 \cdot 49 \%$ & $46 \cdot 36 \%$ & 231 & $11 \cdot 30 \%$ & $35 \cdot 21 \%$ & 269 & $13 \cdot 15 \%$ & $29 \cdot 69 \%$ & 56 & $2 \cdot$ \\
\hline Business & 3 & $0 \cdot 15 \%$ & $2 \cdot 73 \%$ & 45 & $2 \cdot 20 \%$ & $6 \cdot 86 \%$ & 37 & $1 \cdot 81 \%$ & $4.08 \%$ & 11 & $0 \cdot t$ \\
\hline
\end{tabular}




\section{Residence}

\begin{tabular}{|c|c|c|c|c|c|c|c|c|c|c|}
\hline Capital City & 39 & $1 \cdot 91 \%$ & $35 \cdot 45 \%$ & 133 & $6 \cdot 50 \%$ & $20 \cdot 27 \%$ & 185 & $9.05 \%$ & $20 \cdot 42 \%$ & 82 \\
\hline $\begin{array}{l}\text { Divisional } \\
\text { City }\end{array}$ & 28 & $1 \cdot 37 \%$ & $25 \cdot 45 \%$ & 159 & $7 \cdot 78 \%$ & $24 \cdot 24 \%$ & 260 & $12 \cdot 71 \%$ & $28 \cdot 70 \%$ & 57 \\
\hline District Town & 13 & $0.64 \%$ & $11 \cdot 82 \%$ & 226 & $11 \cdot 05 \%$ & $34 \cdot 45 \%$ & 219 & $10 \cdot 71 \%$ & $24 \cdot 17 \%$ & 117 \\
\hline $\begin{array}{l}\text { Upazila (Sub } \\
\text { District) } \\
\text { Town }\end{array}$ & 25 & $1 \cdot 22 \%$ & $22 \cdot 73 \%$ & 106 & $5 \cdot 18 \%$ & $16 \cdot 16 \%$ & 87 & $4 \cdot 25 \%$ & $9.60 \%$ & 48 \\
\hline $\begin{array}{l}\text { Village or } \\
\text { Rural }\end{array}$ & 5 & $0 \cdot 24 \%$ & $4 \cdot 55 \%$ & 32 & $1 \cdot 56 \%$ & $4 \cdot 88 \%$ & 155 & $7 \cdot 58 \%$ & $17 \cdot 11 \%$ & 69 \\
\hline
\end{tabular}

\section{Monthly \\ Family \\ Income}

Less than

26

$1 \cdot 27 \%$

$23 \cdot 64 \%$

117

$5 \cdot 72 \%$

$17 \cdot 84 \%$

272

$13 \cdot 30 \%$

$30 \cdot 02 \%$

96

$4 \cdot 6$

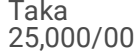

Taka

3

$25,000 / 00$ to

$60,000 / 00$

Over Taka

60,000/00

$37 \quad 1.81 \%$

$33 \cdot 64 \% \quad 263$

$263 \quad 12 \cdot 86 \%$

$40.09 \%$

387

$18.92 \%$

$42 \cdot 72 \%$

151

$7 \because$

Marital

Status

\begin{tabular}{|c|c|c|c|c|c|c|c|c|c|c|c|}
\hline Never Married & 58 & $2 \cdot 84 \%$ & $52 \cdot 73 \%$ & 371 & $18 \cdot 14 \%$ & $56 \cdot 55 \%$ & 611 & $29 \cdot 88 \%$ & $67 \cdot 44 \%$ & 280 & 13 \\
\hline Ever Married & 52 & $2 \cdot 54 \%$ & $47 \cdot 27 \%$ & 285 & $13 \cdot 94 \%$ & $43 \cdot 45 \%$ & 295 & $14 \cdot 43 \%$ & $32 \cdot 56 \%$ & 93 & $4 \cdot 5$ \\
\hline \multicolumn{12}{|l|}{$\begin{array}{l}\text { COVID-19 } \\
\text { Knowledge } \\
\text { Test }\end{array}$} \\
\hline $\begin{array}{l}\text { Poor } \\
\text { Knowledge }\end{array}$ & 35 & $1 \cdot 71 \%$ & $31 \cdot 82 \%$ & 342 & $16 \cdot 72 \%$ & $52 \cdot 13 \%$ & 393 & $19 \cdot 22 \%$ & $43 \cdot 38 \%$ & 153 & $7 \cdot 4$ \\
\hline $\begin{array}{l}\text { Good } \\
\text { Knowledge }\end{array}$ & 75 & $3 \cdot 67 \%$ & $68 \cdot 18 \%$ & 314 & $15 \cdot 35 \%$ & $47 \cdot 87 \%$ & 513 & $25 \cdot 09 \%$ & $56 \cdot 62 \%$ & 220 & 10 \\
\hline
\end{tabular}

Risks of infections were measured with selected questions from practices. Risk score then analyzed with demographic classification and C19KT score to know the diversity of risks.

Binary logistic regression analysis on the basis of selected attitude and practices towards Knowledge has shown Table 05.

Table: 05: Binary logistic regression analysis based on selected attitude and practices towards

knowledge 


\begin{tabular}{|c|c|c|c|}
\hline & Odds Ratio (OR) & 95\% Confidence Intervals & $\mathbf{P}$ \\
\hline Wash hands with soap frequently (no vs yes) & $0 \cdot 391$ & $0 \cdot 228-0 \cdot 671$ & 0.001 \\
\hline Always use mask (no vs yes) & 0.944 & $0 \cdot 737-1 \cdot 209$ & $0 \cdot 649$ \\
\hline Maintain the rules of using mask (no vs yes) & 0.568 & $0 \cdot 456-0 \cdot 709$ & $<0.001$ \\
\hline Meet friends regularly in these days (no vs yes) & $2 \cdot 139$ & $1 \cdot 683-2 \cdot 719$ & $<0.001$ \\
\hline Maintain social distance (no vs yes) & 0.991 & $0 \cdot 735-1 \cdot 337$ & 0.955 \\
\hline Drink tea/coffee from roadside shops (no vs yes) & $0 \cdot 870$ & $0.692-1.094$ & $0 \cdot 234$ \\
\hline Eat snack from roadside shops (no vs yes) & $1 \cdot 152$ & $0 \cdot 857-1 \cdot 548$ & $0 \cdot 349$ \\
\hline Work with sick people or health worker (no vs yes) & $2 \cdot 121$ & $1 \cdot 657-2 \cdot 715$ & $<0.001$ \\
\hline Recent visit of corona virus infected area (no vs yes) & $2 \cdot 500$ & $1 \cdot 374-4 \cdot 545$ & 0.003 \\
\hline Recent meeting with people came from abroad (no vs yes) & $0 \cdot 498$ & $0 \cdot 247-1 \cdot 003$ & $0 \cdot 050$ \\
\hline Corona infection symptoms at work places or near work place (no vs yes) & $0 \cdot 412$ & $0 \cdot 285-0 \cdot 596$ & $<0.001$ \\
\hline Family member(s) caught corona infection (no vs yes) & 0.984 & $0 \cdot 248-3 \cdot 912$ & 0.982 \\
\hline Frequently touching mouth or eyes or nose or all frequently (no vs yes) & $1 \cdot 260$ & $1 \cdot 007-1 \cdot 577$ & 0.043 \\
\hline Risk of being infected with corona virus (low and medium risk vs high and extreme high risk) & 0.651 & $0 \cdot 477-0 \cdot 888$ & 0.007 \\
\hline
\end{tabular}

Selected practice questions that were used in the analysis of risks of infections. These questions were then used to do the multiple binary logistic regression analysis with C19KT scores for identifying the association. First category of each independent variable was the reference category and C19KT score was dependent category (poor knowledge was reference).

Among thirteen selected attitudes/practices and risk score, eight were significantly associated with knowledge. Multiple binary logistic regression analysis on demographic factors and C19KT score towards risk of infections (risk score) has shown in Table 06. Among the independent variables, education levels (OR: $3.164, P=0.001)$, residence (OR: $2 \cdot 056, P=0.03)$ and $C 19 K T$ score (OR: $0.356, P<0.001)$ were significantly associated with risk of infections.

Table 06: Binary logistic regression based on demographic factors towards risks of infections

\begin{tabular}{|c|c|c|c|}
\hline & Odds ratio (OR) & 95\% Confidence Intervals & $\mathbf{P}$ \\
\hline Age & $1 \cdot 058$ & $0 \cdot 789-1 \cdot 419$ & $0 \cdot 708$ \\
\hline \multicolumn{4}{|l|}{ ( 16 to 30 years old vs $30+$ years old) } \\
\hline Gender & $3 \cdot 493$ & $2 \cdot 847-4 \cdot 284$ & 0.000 \\
\hline \multicolumn{4}{|l|}{ (male vs female) } \\
\hline Education & $1 \cdot 405$ & $1 \cdot 059-1 \cdot 865$ & 0.018 \\
\hline \multicolumn{4}{|l|}{ (up to HSC vs above HSC) } \\
\hline Occupation & $2 \cdot 175$ & $1 \cdot 730-2 \cdot 736$ & $0 \cdot 000$ \\
\hline \multicolumn{4}{|l|}{ (unemployed vs employed) } \\
\hline Residence & $0 \cdot 818$ & $0 \cdot 645-1 \cdot 038$ & 0.099 \\
\hline \multicolumn{4}{|l|}{ (village/rural vs urban) } \\
\hline Monthly Family Income & 0.862 & $0 \cdot 697-1 \cdot 065$ & 0.169 \\
\hline \multicolumn{4}{|l|}{ (low and medium income vs high income) } \\
\hline Marital Status & $1 \cdot 227$ & $0 \cdot 964-1 \cdot 563$ & 0.097 \\
\hline \multicolumn{4}{|l|}{ (never married vs ever married) } \\
\hline C19KT Score & 0.789 & $0 \cdot 649-0 \cdot 960$ & 0.018 \\
\hline (poor knowledge vs good knowledge) & & & \\
\hline
\end{tabular}


Multiple binary logistic regression analysis was conducted to find the association of demographic characters and C19KT score with Risk of Infection. The first category of each independent variable was reference category. Risk score was dependent variables and Low and Medium Risk score was the reference category over high and extreme high risk category.

\section{Discussion:}

According to a leaked inter-agency UN memo dated 26 March 2020 [28], due to the odd population densities in Bangladesh, globally recognized modeling techniques and parameter assumptions, the COVID-19 situations can be as worsen and up to 2 million people may die if no fruitful intervention is taken. The message is clear, people of Bangladesh need to know the coping strategy regarding the pandemic and should take necessary measures accordingly. To our best knowledge, this is the first study in Bangladesh regarding KAP investigation towards COVID-19 among Bangladeshi residents. The study results indicate that nearly half of the populations of Bangladesh are not well aware about the COVID-19 and the upcoming hazardous situation as warned by the UN memos. To prevent or reduce the infection rates, people need to get information and act accordingly.

Despite lack of knowledge regarding COVID-19, the Bangladeshi residents are careful to avoid potential problems or dangers of it. Nearly all person washes their hands frequently, nearly three fourth of the people wear masks, around $87.97 \%$ people avoid social gatherings, and nearly one third of the online participants sneezed between their elbows in this pandemic. Due to socioeconomic conditions and other unspecified reasons, very few Bangladeshi people repeatedly washed their hands with soaps and alcohol based sanitizers before, but scenarios have been changed now. The features of KAP towards COVID-19 were evaluated and some demographic determinants associated with KAP were identified. Significant positive association between education levels and C19KT scores make hopes for the better situations. As expected majority of the GKP are from Dhaka Division (27.54\% of GKP), and the lowest GKP was surprisingly observed from Sylhet Division (4.01\%). The reasons behind this perhaps could be the availability and the price of internet. Likewise, people of village/rural (17.11\% of GKP) and Upazila towns (13.81\% of GKP) are less knowledgeable compared to Urban (Capital city: $21 \cdot 03 \%$ of GKP, Divisional cities: $20 \cdot 32 \%$ of GKP, District towns: $27 \cdot 72 \%$ of GKP) for the similar reasons.

In this pandemic situation, attitude and practices towards COVID-19 did depend on the information they got and subsequently acted accordingly. Studies [18]showed that coronavirus can be transmitted through cloths and shoes. However, people of Bangladesh have less concern about it. Most of the people did not clean their mobile phone (60.00\%) or touched mobile phone with unwashed hands (50.86\%). Due to massive spreading news, most of the people are avoiding for going to corona affected areas (97.02\%) and also avoiding to come in touch of the people who are coming from abroad or corona affected areas (98.09\%). Total 10 respondents informed that their family members were infected with coronavirus that made them in extreme risk categories of infection. People who are above 55 years olds are in high risk categories. Among thirteen respondents, five (38.46\% of this age group) were in extreme high risk categories and seven (53.85\% of this age group) were in high risk group.

The present study indicates that $12 \cdot 03 \%$ people did not maintain social distances as well as $32 \cdot 27 \%$ regularly met with friends and colleagues and $24 \cdot 45 \% \%$ did not wear masks when went outside. These potentially precarious activities were associated to males, students, marital status, residents, and poor knowledgeable people regarding COVID-19. As advised by outcomes from earlier studies about age and gender patterns of risk-taking manners [29], men and late adolescents are more prone to get involved in risk-taking performances. The strength of this study lies in its initiation of this online survey at the early stage of the COVID-19 outbreak in Bangladesh. Comparing the recent work on KAP towards COVID19 of Chinese population [30], the knowledge of Bangladeshi population is low and the attitude and practices were also not that level because of socioeconomic and health care systems.

The findings of the present study suggest that half of the Bangladeshi peoples have good knowledge, however, their attitude and practices towards COVID-19 during the pandemic were not impressive. In addition, COVID-19 knowledge and practices are associated with demographic characteristics. Proper health education and mass awareness programs would be helpful for improving attitudes and maintaining safe practices. Appropriate preventive measures, healthy practices, and instructions must be strictly implemented by the government with the help of concerned agencies and organizations. Moreover, the WHO and UNICEF guidelines must comply by all the section of the people. The outcomes of the study would be very much useful for public health policy-makers and health workers. Moreover, preventive and coping strategy would be enriched along with overall health education updates from the elementary level of education. Hopefully, under the combined efforts of Bangladesh Government and the people of Bangladesh, the country certainly will win the battle against COVID-19 pandemic.

\section{Limitation of the study}

Due to limited access to internet and other logistic support, it was not possible to bring a large number of people of the country under this study.

\section{Declarations}




\section{Contributors}

TH and MMR conceived the study with input from MMRB and SA. TH and MMR developed questionnaires and study design. TH led the project regarding volunteer recruitments, data collections to writing with the help of MMRB and SA. MMR led the analysis of individual-case data and estimation of the onset-tooutcome distributions, with input from TH, KMH, MMRB, AA and MRI. TH coordinated management of the team, including the data collection, analysis and processing. TH and MMR produced the first draft of the manuscript, KMH did put efforts regarding writings and corrections of the manuscripts; SHC, $A A$ and $\mathrm{KMH}$ added additional points in discussions. TH, KMH, MRI, AA and MMR finalized the manuscripts after necessary corrections and obtaining suggestions from all authors. MMR supervised all the works from the beginning to the end. All authors did read and agreed unanimously to submit the manuscripts.

\section{Declaration of interests}

This study has not yet received any funds from any institute, organizations or government. All authors declare that there is no conflict of interests among them.

\section{Acknowledgments}

The authors gratefully acknowledge the efforts provided by the volunteers and the respondents who participated in this online survey.

\section{Ethics}

This work is approved by the Ethics Committee of Bangladesh University of Health Sciences. In addition, data from all respondents were collected through an online survey where they had to made consent by clicking "yes" to the questions mentioning they understood the theme of study and are willing to participate in this survey without being forced by anyone.

\section{References}

1. "Naming the coronavirus disease (COVID-19) and the virus that causes it". https://www.who.int/emergencies/diseases/novel-coronavirus-2019/technicalguidance/naming-the-coronavirus-disease-(covid-2019)-and-the-virus-that-causes-it. Retrieved 28 February 2020.

2. Hui DS, Azhar E, Madani TA, et.al. The continuing 2019-nCoV epidemic threat of novel coronaviruses to global health-The latest 2019 novel coronavirus outbreak in Wuhan, China. Int J Infect Dis. 2020; 91: 264-66.

3. "WHO Director-General's opening remarks at the media briefing on COVID-19". https://www.who.int/dg/speeches/detail/who-director-general-s-openingremarks-at-the-media-briefing-on-covid-19--20-march-2020. World Health Organization (WHO) (Press release). Retrieved 26 March2020.

4. COVID-19 coronavirus pandemic. http://www.winfo/coronavirus. Online information portal. Retrieved 8April 2020.

5. Zhang H, Penninger JM, Li Y, Zhong N, Slutsky AS. Angiotensin-converting enzyme 2 (ACE2) as a SARS-CoV-2 receptor: molecular mechanisms and potential therapeutic target. Intensive Care Medicine. 2020; 46 (4): 586-590.

6. "Coronavirus Disease 2019 (COVID-19) Symptoms".https://www.cdc.gov/coronavirus/2019-ncov/symptoms-testing/symptoms.html. Centers for Disease Control and Prevention. United States. Retrieved 27Marchl 2020.

7. Wei XS, Wang X, Niu YR et.al. (26 February 2020). Clinical Characteristics of SARS-CoV-2 Infected Pneumonia with Diarrhea. Rochester, NY. SSRN2020; 3546120

8. Huang C, Wang Y, Li X, et al. Clinical features of patients infected with 2019 novel coronavirus in Wuhan, China. TheLancet 2020; 395(10223): 497-506.

9. Hopkins C. "Loss of sense of smell as marker of COVID-19 infection". Ear, Nose and Throat surgery body of United Kingdom. Retrieved 28 March 2020.

10. lacobucci, G (2020). "Sixty seconds on ... anosmia". 2020; 368: m1202.

11. "Q\&A on coronaviruses". World Health Organization (WHO). https://www.who.int/news-room/q-a-detail/q-a-coronaviruses. Retrieved 27 March 2020.

12. "Coronavirus Disease 2019 (COVID-19)". Centers for Disease Control and Prevention. https://www.cdc.gov/coronavirus/2019-ncov/index.html. 27 March 2020.

13. Gu J, Han B, Wang J. (27 February 2020). COVID-19: Gastrointestinal manifestations and potential fecal-oral transmission. (Article in Press)

14. Hamming I, Timens W, Bulthuis M L C, Lely AT, Navis G J, Goor H. van . Tissue distribution of ACE2 protein, the functional receptor for SARS coronavirus. A first step in understanding SARS pathogenesis. The Journal of Pathology. 2004; 203 (2): 631-637.

15. "CoV2020". GISAID EpifluDB. Archived (https://web.archive.org/web/20200112130540/https://platform.gisaid.org/epi3/start/CoV2020) from the original. Retrieved 27 March 2020.

16. Zhu N, Zhang D, Wang W et al. A Novel Coronavirus from Patients with Pneumonia in China, 2019. The New England Journal of Medicine. $2020 ; 382$ (8): 727-733.

17. Lauer SA, Grantz KH, Bi Q, et.al. The Incubation Period of Coronavirus Disease 2019 (COVID-19) From Publicly Reported Confirmed Cases: Estimation and Application. Annals of Internal Medicine. 
18. van Doremalen N, Bushmaker T, Morris DH, et al. Aerosol and Surface Stability of SARS-CoV-2 as Compared with SARS-CoV-1. The New England Journal of Medicine. 2020

19. "Advice for public". World Health Organization (WHO). https://www.who.int/emergencies/diseases/novel-coronavirus-2019/advice-for-public.Retrieved 27 March 2020.

20. "Guidance on social distancing for everyone in the UK". GOV.UK. https://www.gov.uk/government/publications/covid-19-guidance-on-social-distancingand-for-vulnerable-people/guidance-on-social-distancing-for-everyone-in-the-uk-and-protecting-older-people-and-vulnerable-adults.Retrieved 25 March 2020.

21. Mahtani S, Berger M, O'Grady S, lati M. Hundreds of evacuees to be held on bases in California; Hong Kong and Taiwan restrict travel from mainland China". The Washington Post. Retrieved 22 March 2020.

22. "Overall total population" - World Population Prospects: The 2019 Revision"(xslx). population.un.org (custom data acquired via website). United Nations Department of Economic and Social Affairs, Population Division. Retrieved 19 March 2019.

23. Data Archived 4 September 2011 at the Wayback Machine. Census - Bangladesh Bureau of Statistics. Retrieved 19 March 2019.

24. Ajilore K, Atakiti I, Onyenankey K. College students' knowledge, attitudes and adherence to public service announcements on Ebola in Nigeria: Suggestions for improving future Ebola prevention education programmes. Health Education Journal. 2017;76:648-60

25. Tachfouti N, Slama K, Berraho M, Nejjari C. The impact of knowledge and attitudes on adherence to tuberculosis treatment: a case-control study in a Moroccan region. Pan Afr Med J. 2012;12:52

26. Person B, Sy F, Holton K, Govert B, Liang A. National Center for Inectious Diseases SCOT. Fear and stigma: the epidemic within the SARS outbreak. Emerg Infect Dis. 2004;10:358-63

27. Tao N. An analysis on reasons of SARS-induced psychological panic among students. Journal of Anhui Institute of Education. 2003;21:78-9

28. "Two million could die in Bangladesh from Coronavirus, warns UN". The South Asian Monitor. https://south-asian-monitor.com/en/bangladesh/twomillion-could-die-in-bangladesh-from-coronavirus-warns-un. Retrieved 30 March 2020.

29. Duell N, Steinberg L, Icenogle G, et al. Age Patterns in Risk Taking Across the World. J Youth Adolesc. 2018;47:1052-72

30. Zhong BL, Luo W, Li HM, et.al. Knowledge, attitudes, and practices towards COVID-19 among Chinese residents during the rapid rise period of the COVID19 outbreak: a quick online cross-sectional survey. Int J Biol Sci. 2020; 16(10): 1745-1752.

\section{Supplementary Files}

This is a list of supplementary files associated with this preprint. Click to download.

- Supplementary.docx 